\title{
Minimization of Average Power Consumption in 3 Stage CMOS Ring Oscillator based on MSFLA, Fuzzy- MSFLA, GA, and Fuzzy-GA
}

\author{
Farshid Keivanian \\ Master of Science in Electronics Engineering \\ Department of Electrical and Computer Engineering \\ University of Birjand, South Khorasan, Birjand, Iran
}

\begin{abstract}
This article is based on the application of heuristic algorithms to minimize the average power consumption in a VLSI circuit. The idea is to find the optimum layout and temperature for a 3 stage ring oscillator with minimal dynamic average power. The objective function is the same as average power (Pavg) of 3 stage ring oscillator with 6 CMOS inverters that depends on the temperature and the two different group of channel widths for NMOSs and PMOSs. (W1=W3=W5 and W2=W4=W6). These parameters make a three dimensional search space which is explored by search agents of algorithms. Motivated by the convergence of Modified Shuffled Frog Leaping Algorithm (MSFLA), Genetic Algorithm (GA) and the link of MATLAB with HSPICE Software the minimized average power of 3 stage ring oscillator is obtained. Based on MSFLA, Fuzzy-MSFLA, GA, and Fuzzy-GA algorithms the best resulting for Pavg in $0.18 \mu \mathrm{m}$ Technology and the supply voltage of $5 \mathrm{v}$ is $1.19 \mu \mathrm{W}$ based on Fuzzy-MSFLA.
\end{abstract}

\section{General Terms}

Heuristic Algorithms, Applied Soft Computing, Fuzzy Logic, Optimization of VLSI circuits.

\section{Keywords}

3 stage CMOS ring oscillator, minimal average power, optimum layout and temperature, MSFLAF, Fuzzy-MSFLA, GA, and Fuzzy-GA.

\section{INTRODUCTION}

Oscillators are used for synchronizing computation in a digital system, timing the sampling in a data converter, carrier synthesis and LO in RF systems [1]. The ring oscillators are widely used in integrated circuits. They are being used be semiconductor founders to monitor the power dissipation, delay, and jitter of fabricated CMOS inverters. On the basis of the result of measured frequency pattern the IC is accepted or rejected. Ring oscillators also occupies less chip area as they do not have inductor as compared to LC tank oscillators [2]. The application of oscillators ranges from clock generation in microprocessor to carrier synthesis in cellular telephones requiring vastly different oscillator topologies and performance parameter [3]. Recently the explosive growth in wireless communication and the advances in complementary metal oxide semiconductor (CMOS) technology made it possible to implement high frequency oscillator with CMOS technology (MR20768). The oscillator are required to be tunable over a relatively wide frequency rang. The tenability is usually obtained by variable voltage and hence comes the name Voltage Controlled Oscillator (VCO). VCOs are key component in frequency synthesizer for Radio Frequency (RF) wireless application [4]. Voltage Controlled Oscillators
(VCOs) constitute a critical component in many RF transceivers and are commonly associated with signal processing tasks like frequency Selection and signal generation. There are two types of VCOs such as I) Waveform oscillators: ring oscillator topology and relaxation oscillator (which has poor phase noise performance) II) Resonant oscillators: LC tank oscillator topology and Crystal oscillator (neither integrated nor tunable) [2]. The ring oscillator topology has the ability of easy integration with the absence of passive elements which reduces the die area. This property changes the ring oscillators to the favorite kind of oscillators in communication systems [5,6,7]. In this paper the heuristic algorithms such as Modified Shuffled Frog Leaping Algorithm (MSFLA) [8,9], Fuzzy-MSFLA, GA, and FuzzyGA are employed to find the best channel widths and temperature in which the 3 stage ring oscillator consumes the lowest average power.

\section{THREE STAGE CMOS RING OSCILLATOR}

The ring oscillator which is proposed in this article is comprised of three delay stages, with the output of the last stage fed back to the input of the first. To provide the DC inversion, an odd number of stages must be used. To see why 3 stage ring circuit will oscillate, assume that the output of the first inverter is a ' 0 '. Therefore, the output of the Nth (3th) inverter, where $\mathrm{N}(\mathrm{N}=$ "3") is odd, must also be ' 0 '. However, this output is also the input to the first inverter, so the first inverter's output must switch to a ' 1 '. By the same logic, the output of the last inverter will eventually switch to a ' 1 ', switching the output of the first inverter back to ' 0 '. This process will repeat indefinitely, resulting in the voltage at each node oscillating [10]. The 3 stage CMOS ring oscillator using single ended inverters is shown in Figure 1 and 2.

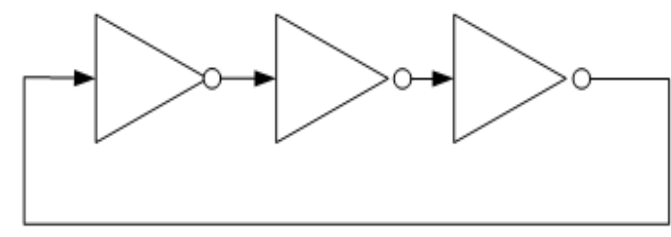

Fig 1: The 3 Stage Ring Oscillator using single ended inverters 


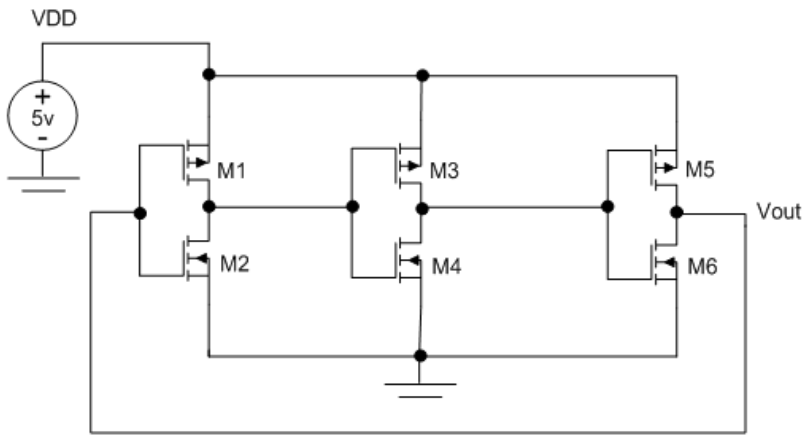

Fig 2: The 3 Stage CMOS Ring Oscillator

\section{HEURISTIC ALGORITHMS: MSFLA AND GA}

In the past few decades, there has been widespread interaction between researchers seeking various evolutionary computation methods to seek the best solutions to a given function. The Evolutionary Algorithms are developed by mimicking or simulating processes found in nature and mainly includes Genetic Algorithms, Mimetic Algorithms, Particle Swarm Optimization, Ant Colony Optimization, and Shuffled Frog Leaping Algorithm (SFLA) [11]

\subsection{The objective Function in this Article}

In this paper the objective function is the average power of a 3 stage ring oscillator. This article is based on minimization work that the single objective of average power is defined at first and it becomes minimized based on heuristic algorithms. The objective function is shown in Equation 1.

Cost Function $\triangleq \mathrm{P}_{\text {avg }}$ (Average Power)

Pavg is the switching (dynamic) consumption average power in the maximum operating frequency in which the oscillator works. This value is calculated by HSPICE software. In fact HSPICE is the fitness evaluator for heuristic algorithms used in this article. The HSPICE command for measuring average power within a specified time is shown in Relation 2. The Relation 3 shows the dependency of Pavg to power supply ("VDD") and load capacitance ("C $\mathrm{C}_{\text {Load }}$ ) and operating frequency ("f") [12].

.MEAS tran avgpower Avg Power from $=200 \mathrm{p}$ to $=200 \mathrm{n}$

$P_{\text {avg }}=\mathrm{V}^{2}$ DD.Cload. $f$

$f=\frac{1}{2 * N * t p} \quad: \quad \mathrm{N}=3, \mathrm{t}_{\mathrm{p}}=\frac{t_{P H L}+t_{P L H}}{2}$

$t_{\mathrm{p}} \propto \frac{2 C_{L}(1-\alpha) V_{D D}}{\mu_{n} C_{o x}\left(\frac{W}{L}\right)\left(V_{D D}-V_{t h}\right)^{2}}+\frac{C_{L}}{\mu_{n} C_{o x}\left(\frac{W}{L}\right)\left(V_{D D}-V_{t h}\right)} \ln \left(\frac{2 \alpha-\beta}{\beta}\right)$

$0<\alpha<1, \alpha<\beta$

$\mu_{n}=\mu_{0} e^{-\left(\frac{E a}{K b . T}\right)}$

$\rightarrow P_{\text {avg }} \propto W$ and $T$

$\mathrm{N}$ is the number of inverters which is 3 here ("N=3"), and tp is propagation delay of each inverter and the operating frequency is shown in Relation 4. The Relation 5 shows that channel widths ("W") have impact on propagation delay [13]. Therefore $\mathrm{W}$ has impact on the operating frequency and finally on the average power, Pavg. Also based on relation 6 by increasing temperature $\mathrm{T}$ the mobility of electrons, $\mu \mathrm{n}$ increases [14]. The effect of temperature $T$ and channel widths $\mathrm{W}$ on average power, Pavg are shown in Relation 7. Thus by changing the parameters of channel widths (W) and temperature $(\mathrm{T})$ the average power consumption (Pavg) varies. In this article all parameters such as NMOS and PMOS channel widths and temperature are defined as independent variables that make a search space in which the optimum solution (value) for NMOS channel widths (W1=W3=W5), PMOS channel widths (W2=W4=W6), and the temperature (T) is found. These solutions meet the least average power value. The pseudo code for average power is illustrated in Box 1.

Box 1. The Pseudo-Code of Objective Function which is defined in this Article for MSFLA and GA Algorithms

- Open the input netlist file ('T3b.sp')

- Seek the NMOSs, PMOSs' channel widths and temperature and allocate the positions of search agents to them ("Assign the Position(1) to W1, W3, W5, Position(2) to W2, W4, and W6, and Position(3) to Temperature value")

- Run HSPICE as a fitness evaluator with input Netlist File ('T3b.sp')

- Read data from output file ('T3b.lis') and seek the average power ('avgpower') from the output file ('T3b.lis') and return it to the main program that has called this objective function

\subsection{Shuffled Frog Leaping Algorithm (SFLA)}

The Shuffled Frog Leaping Algorithm (SFLA) which is firstly proposed by Eusuff and Lansey. The overall structure in SFLA is shown in Figure 3 which is the same as structure of shuffled complex evolution (SCE-UA) [15-18]. The flowchart of SFLA in Figure 4 is similar to the operation of SCE-UA except that the frog leaping algorithm (FLA) is used as local search in SFLA while in SCE-UA for local search the competitive complex evolution (CCE) is used. One of differences between FLA and CCE is that the parameter of maximum search step, Smax is defined in FLA to limit the length of search step because it is randomly produced. The vector calculations for producing solutions in standard SFLA are in Relations 8, 9, and 10 and they are shown in Figure 5.

$S=\operatorname{round}(\operatorname{rand} \times C(X-P w)), X \in\left\{P_{B}, P_{X}\right\}, C>0$

$|S|=\min \left(|S|, S_{\max }\right)$

$U_{q}=P w+S$

The Relation 8 shows that $\mathrm{X}$ is either the best solution in complex, PB, or the best global solution, PX, and the deference of $X$ and the worst solution of complex, Pw, the search step of $\mathrm{S}$ is obtained. The parameter $\mathrm{C}$ commonly equals to $2(\mathrm{C}=2)$. The maximum search step length, Smax is obtained in Relation 9 and finally the new vector of solution $\mathrm{Uq}$ is obtained in 10. In Figure 3 the allocation of population to complexes, selection of $\mathrm{q}$ members of complexes with triangular distribution of pi , modification or improvement of the last member in each of complexes, placement of improved complex in the main population and sorting the populations in the new complex, then repeat the entire process for all complexes. The results of global search are used in local search. Figure 5 shows the sampling process in SFLA. 


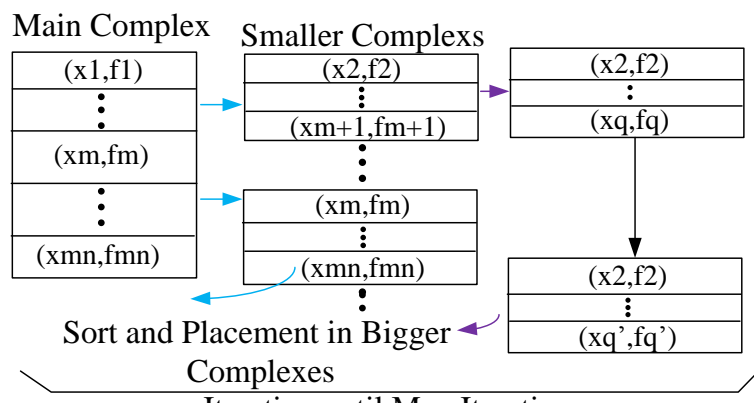

Iteration until Max Iteration

Fig 3: The overview of SFLA

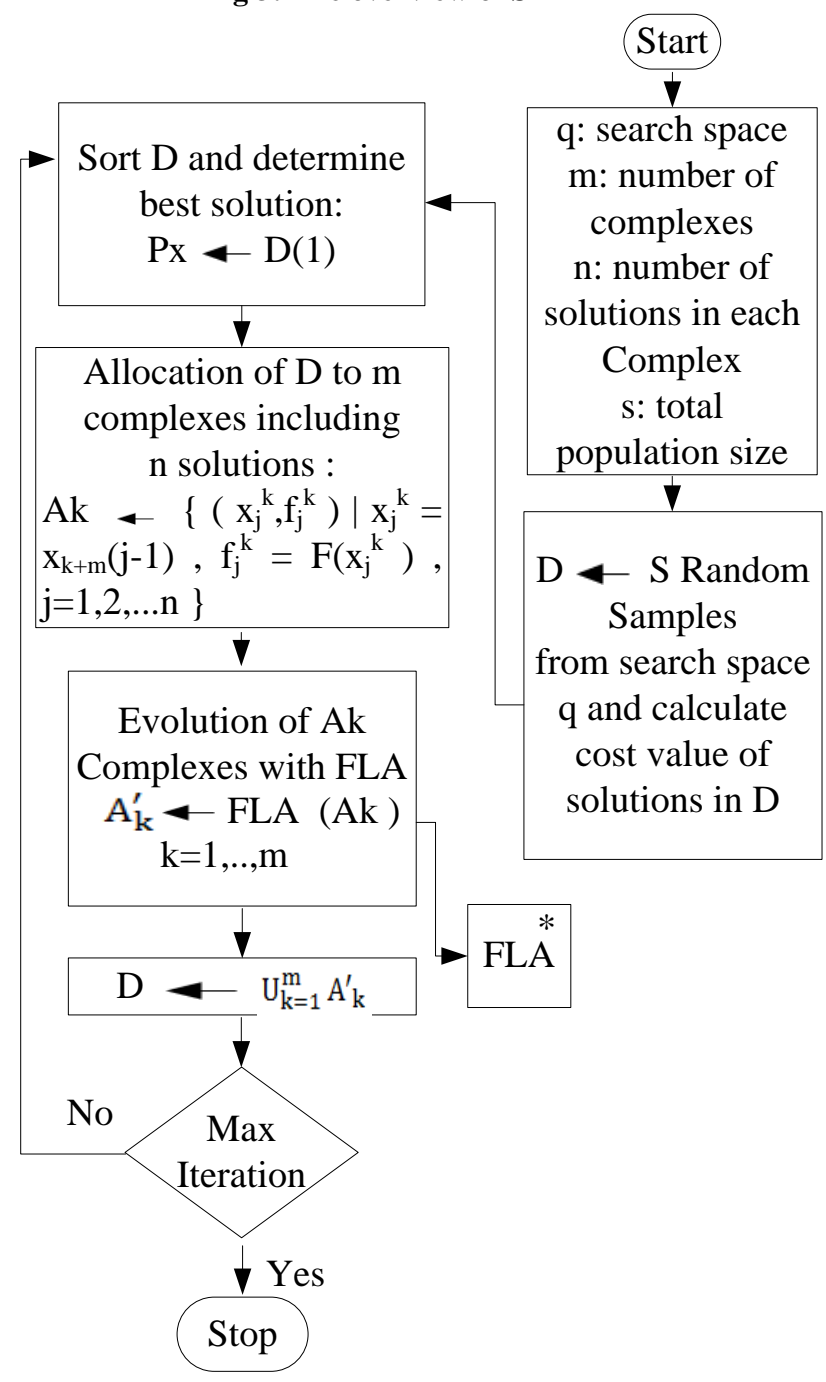

Fig 4: The Flowchart of SFLA

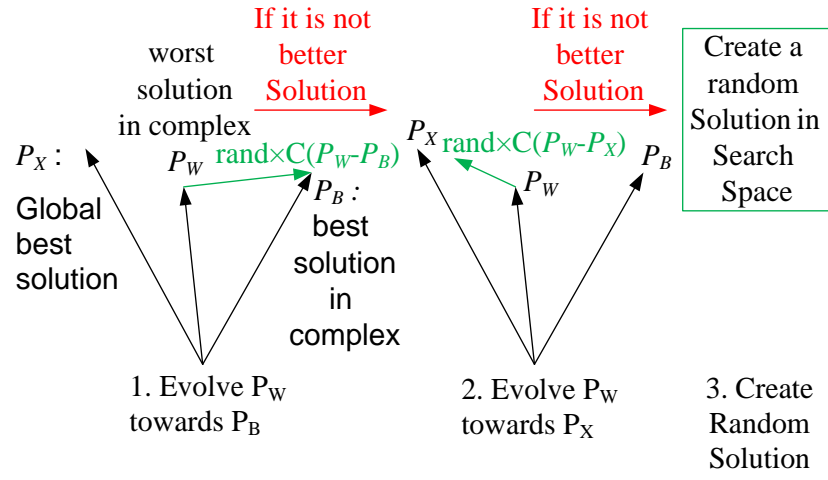

Fig 5: The process of sampling in local search space consists of frog leaping steps in standard SFLA

\subsection{Modified Shuffled Frog Leaping Algorithm (MSFLA)}

All modified versions of SFLA (MSFLAs) maintain the general structure of SFLA. Some of recent articles propose some changes in parameters like $\mathrm{n}$, number of solutions in each complex and others that lead to slight improvement. In other modified versions of SFLA (MSFLA) the parts of local search and the process of sampling in the search space are modified [19-21].

\subsubsection{Modified Shuffled Frog Leaping Algorithm with Cognitive Behavior}

The Modified SFLA (MSFLA) with cognitive behavior is partly based on PSO algorithm as there are two components of individual factor $\mathrm{C}_{1}$ and the social factor $\mathrm{C}_{2}$ in both of them. In Relation 11 the social factor $\mathrm{C}_{2}$ is multiplied by the difference of solution $\mathrm{W}$ with the best solution in complex , $\mathrm{P}_{\mathrm{X}}$ or with the best global solution in population, $\mathrm{P}_{\mathrm{B}}$. The individual factor $C_{1}$ is multiplied by the difference of the solution $\mathrm{W}$ and the best solution of complex $\mathrm{P}_{\mathrm{W} \text {,best }}$ that is experienced. Parameters $r_{1}$ and $r_{2}$ are randomly produced in MATLAB and have uniform probability distribution. For applying the maximum step length the way of standard SFLA is also used for Relation 12. The candidate solution is made by adding the search step with the position of solution $\mathrm{S}$ in Relation 13.

$$
\begin{aligned}
& S=\operatorname{round}\left(r_{1} c_{1}\left(P_{w, \text { best }}-P_{w}\right)+r_{2} c_{2}\left(X-P_{w}\right)\right) \\
& r_{1}, r_{2} \sim U(0,1), X \in\left\{P_{B}, P_{X}\right\} \\
& S=\min \left(S, S_{\text {max }}\right) \\
& U_{q}=P w+S
\end{aligned}
$$

After implementation of Modified SFLA with cognitive behavior on the objective function which is defined the average power consumption in 3 stage ring oscillator the Figures 6 is obtained. It shows the convergence of algorithm with the least average power of $2.4 * 10^{-6}$ watt for the specific values of temperature, NMOS channel widths, and PMOS channel widths that all are shown in Table 1. 


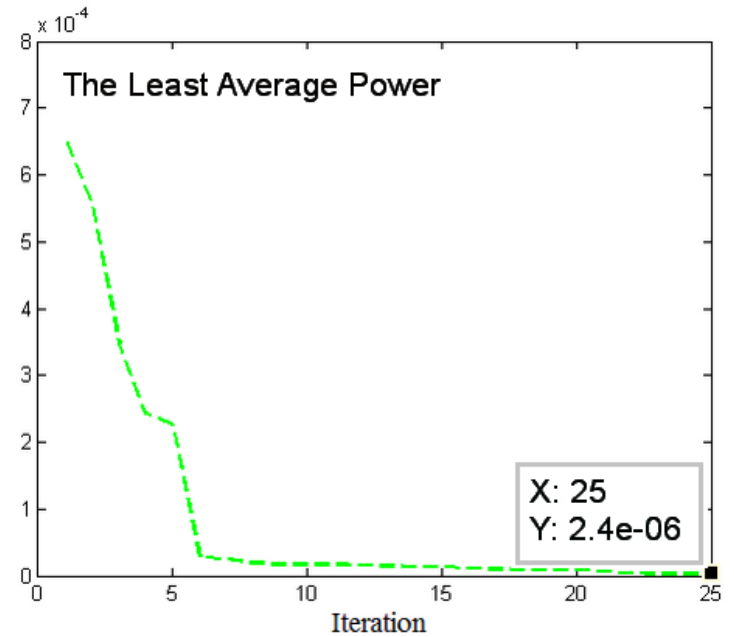

Fig 6: Average Power $=2.4 * 10-6$ Watt, VDD $=5 \mathrm{v}$, Temperature $=46.5339175361742^{\mathrm{c}}$

Table 1. Channel Widths and Temperature based on Modified SFLA with cognitive behavior

\begin{tabular}{|c|c|c|}
\hline $\begin{array}{c}\text { W1=W3=W5 } \\
(\boldsymbol{\mu m})\end{array}$ & $\begin{array}{c}\text { W2=W4=W6 } \\
(\boldsymbol{\mu m})\end{array}$ & $\begin{array}{c}\text { Temperature } \\
(\text { centigrade })\end{array}$ \\
\hline 1.6657126869238 & 0.63834494370498 & 46.533917536174 \\
\hline
\end{tabular}

The Relation 14 shows the total size of population in S. Also the maximum search step, $S_{\max }$ is obtained through the Relation 15.

$S=(\max V a r s-\operatorname{minVars})$

$S_{\max }=0.45 \times(\max V a r s-\operatorname{minVars})$

The probability of selection of the members of the complex A to be evolved is shown in Relation 16.

$p_{i} \leftarrow \frac{2(n+1-i)}{n(n+1)}, i=1, \ldots, n$

The pseudo code for the function of evolve_towrads_pxpb is shown in Box 2. These evolutions are based Relations 11-13.

\section{Box 2. Pseudo-Code of Evolve_Toward_PxPB Function}

- Set parameters $\mathrm{C} 1=1, \mathrm{C} 2=2$

- Calculate the search step : $\mathrm{S}=\max (\min (\mathrm{C} 1 *$ rand $*$ (Pw.Pbest.X - Pw.X) + ... C2 * rand *(Pb.X-Pw.X),Smax),Smax) (based on relations 11,12 )

- Add the search step to the position of the solution to produce a new solution : (based on relation 13)

- Return the produced new random solution to the main program that has called this function

The improvements of SFLA are colored in purple within pseudo codes in Box 3

\section{Box 3. Pseudo-Code of MSFLA with Cognitive Behavior}

- Set the SFLA parameters for the problem of 3 Stage Ring Oscillator, $\mathrm{nVars}=3$ (Number of Variables), $\max V$ ars $=$ [2 25 50] as upper band of NMOS W, PMOS W, and temperature, minVars $=\left[\begin{array}{lll}0.2 & 0.2 & 1\end{array}\right]$ as lower band of NMOS W, PMOS W, and temperature, Smax $=0.45 *(\max V$ ars-minVars $)$, Cost Function=Objective Function ("defined in Box1"), $m=5$ (number of complexes), $\mathrm{n}=5$ (number of solutions in each complex), $\mathrm{S}=\mathrm{m} * \mathrm{n}, \mathrm{q}=4$ (number of members in subcomplex), $\mathrm{Ns}=2$ (number of shuffling), $\mathrm{Nt}=1$ (number of iteration for evolution), nIter $=10$ (total number of iteration for main loop, VTR $=0$ (the lowest value to reach)

- Generate Initial Population, Evaluate the cost value, make a memory for the best solution in each complex by now

-Do While iteration number (it) < Maximum Iteration (nIter) and best cost $>$ VTR

- Sort population and $\mathrm{P}_{\mathrm{X}}$ is determined. $\left(\mathrm{P}_{\mathrm{X}}=\operatorname{pop}(1)\right)$

- For $\mathrm{k}=1$ to $\mathrm{m}$

- Allocation of the population into $\mathrm{k}$ complexes , $\mathrm{A}_{\mathrm{k}}$ (Complex Evolution : FLA)

- For $\mathrm{t}=1$ to $\mathrm{Nt}$

- Select q members from $A_{k}$, sort them and allocate $q$ members of $A_{k}$ into sub-complex of $B$ based on probability distribution $\mathrm{Pi}$ (relation 16)

$$
\begin{aligned}
& \text { - For } \mathrm{j}=1 \text { to Ns } \\
& \text { - Sort B and determine } \mathrm{P}_{\mathrm{B}}, \mathrm{P}_{\mathrm{W}} \\
& \text { - Evolve } \mathrm{Pw} \text { towards } \mathrm{P}_{\mathrm{B}}(\mathrm{PB} \text { : The best global } \\
& \text { solution) } \\
& \text { and produce new solution } \mathrm{r} \text { (Call } \\
& \text { evolve_towrads_pxpb function) } \\
& \text { - if solution } \mathrm{r} \text { is not within minVars, maxVars then } \\
& \text { - Evolve } \mathrm{Pw} \text { towards } \mathrm{P}_{\mathrm{X}} \text { (The best position in } \\
& \text { complex by now, based on memory content) and } \\
& \text { produce new solution c (Call } \\
& \text { evolve_toward_pxpb function) }
\end{aligned}
$$

- if solution $\mathrm{c}$ is not within minVars, maxVars then

- Create new random solution z

- Update the $\mathrm{P}_{\mathrm{B}}$ and Update $\mathrm{P}_{\mathrm{X}}$

- End Ns

-End Nt

- Replace sub complexes of B into Ak, sort Ak.Cost

- Replace complex into Population

\section{- End Do}

- Plot Best Costs

\subsection{Fuzzy Modified Shuffled Frog Leaping Algorithm with Cognitive Behavior (Fuzzy-MSFLA)}

By controlling the parameter $\mathrm{C}_{1}$ (individual factor) and $\mathrm{C}_{2}$ (global factor) the performance of MSFLA is improved. The Fuzzy Rules for this purpose are shown in Table 2 
Table 2. Fuzzy Rules in Modified SFLA with cognitive behavior (Fuzzy-MSFLA)

\begin{tabular}{|c|c|}
\hline If & Then \\
\hline $\begin{array}{c}\text { Iternormalized is Low and } \\
\text { Bestnormalized is High }\end{array}$ & $\begin{array}{c}\mathrm{C} 1 \text { is Low and C2 is } \\
\text { High }\end{array}$ \\
\hline $\begin{array}{c}\text { Iternormalized is Medium and } \\
\text { Bestnormalized is Medium }\end{array}$ & $\begin{array}{c}\mathrm{C} 1 \text { is Medium and } \\
\text { C2 is Medium }\end{array}$ \\
\hline $\begin{array}{c}\text { Iternormalized is High and } \\
\text { Bestnormalized is Low }\end{array}$ & $\begin{array}{c}\text { C1 is High and C2 is } \\
\text { Low }\end{array}$ \\
\hline $\begin{array}{c}\text { Iternormalized is High and } \\
\text { Bestnormalized is High }\end{array}$ & $\begin{array}{c}\text { C1 is Low and C2 is } \\
\text { High }\end{array}$ \\
\hline $\begin{array}{c}\text { Iternormalized is Low and } \\
\text { Bestnormalized is Low }\end{array}$ & $\begin{array}{c}\text { C1 is High and C2 is } \\
\text { Low }\end{array}$ \\
\hline
\end{tabular}

The best result for minimization of average power based on Fuzzy-MSFLA is shown in Figure 7 and Table 3.

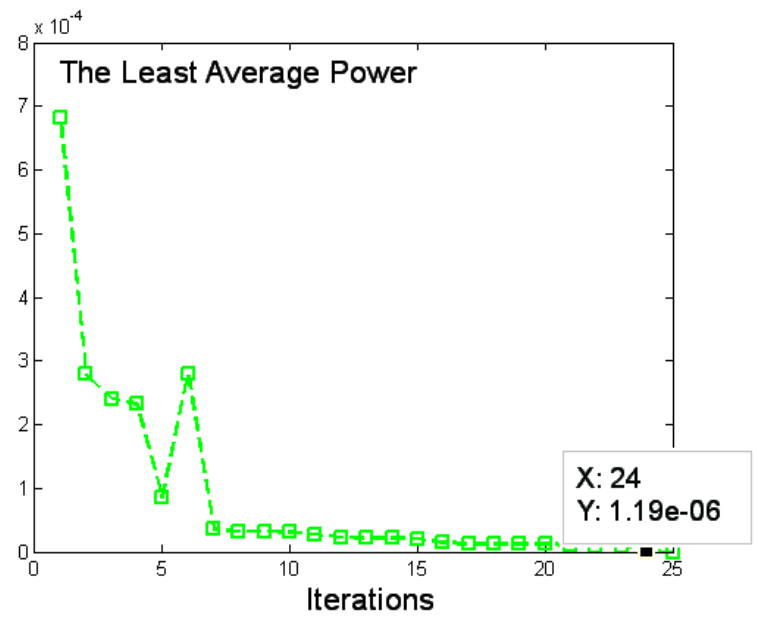

Fig 7: Average Power $=1.19 * 10^{-6}$ Watt, VDD $=5 \mathrm{v}$, Temperature $=46.5339175361742 \mathrm{c}$

Table 3. Channel Widths and Temperature based on Fuzzy Modified SFLA with cognitive behavior (Fuzzy-MSFLA)

\begin{tabular}{|c|c|c|}
\hline $\mathbf{W 1}=W 3=W 5(\boldsymbol{\mu m})$ & $\mathbf{W} 2=W 4=W 6(\boldsymbol{\mu m})$ & $\begin{array}{c}\text { Temperature } \\
\text { (centigrade) }\end{array}$ \\
\hline 0.297848989765905 & 0.322296653819349 & 29.1031851838616 \\
\hline
\end{tabular}

\subsection{Genetic Algorithm (GA)}

In Genetic Algorithm the search agents are the chromosomes. The best chromosomes have the best objective function. Based on pseudo in Box 4 the program worked and the least average power which obtained by GA was shown in Figure 8 .

\section{Box 4. GA Pseudo-Code in this Article}

-Define the problem of 3 Stage Ring Oscillator, CostFunction=Objective Function ("in Box2"), nVar=6, VarMin: 0.2 for channel widths and 1 for temperature, VarMax: 2 for channel widths and 50 for temperature,
MaxIt=10, nPop=4 (Population Size) , pc $=0.5$ (Crossover Percentage), $\mathrm{pm}=0.5$ (Mutation Percentage)

- Generate Initial Population, Evaluate, Sort, and place them in the Best Solutions

-Do While iteration number (it) < Maximum Iteration (MaxIt)

- Calculate Selection Probabilities

- Select Parents indices and apply crossover and evaluate off springs, in Crossover Operator

- Select Parents indices and apply mutation and evaluate Mutant, in Mutation Operator

- Create Merged Population, sort population

- Update and store the best solution and Cost that ever found

\section{End Do}

Plot Results

GA is firstly implemented in MATLAB software and the optimum layout for the 3 stage CMOS ring oscillator is obtained with power supply of $\mathrm{Vs}=5 \mathrm{v}$ and the temperature of $25^{\mathrm{c}}$. The channel widths ("BestSol.Positions") for NMOSs and PMOSs are shown in Table 4 also the convergence of power dissipation is illustrated in Figure 8.

Table 4. Channel Widths ("BestSol.Positions")

\begin{tabular}{|c|c|}
\hline $\mathbf{W 1}=W 3=W 5(\boldsymbol{\mu m})$ & $\mathbf{W} 2=W 4=W 6(\boldsymbol{\mu m})$ \\
\hline 1.50897150624236 & 0.640468867234475 \\
\hline
\end{tabular}

For implementation of algorithms the attention is focused to the fact that the channel widths of PMOSs have the same values and the channel widths of NMOSs have the identical values.

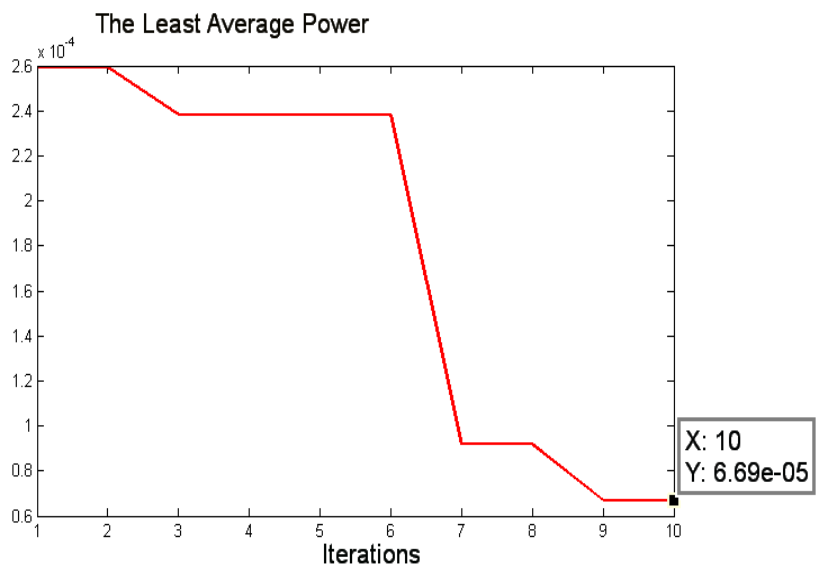

Fig 8: Average Power $=6.69 * 10^{-5}, \mathrm{VDD}=5 \mathrm{v}$, Temperature $=25^{\mathrm{c}}$

The values of channel width that were shown in Table 4 , remain unchanged in the input netlist file ("V3.sp") in HSPICE Software. The optimization is performed to find the best solution for the temperature that for which the average power is more reduced. The convergence is shown in Figure 9 with the optimum temperature. 


\section{The Least Average Power}

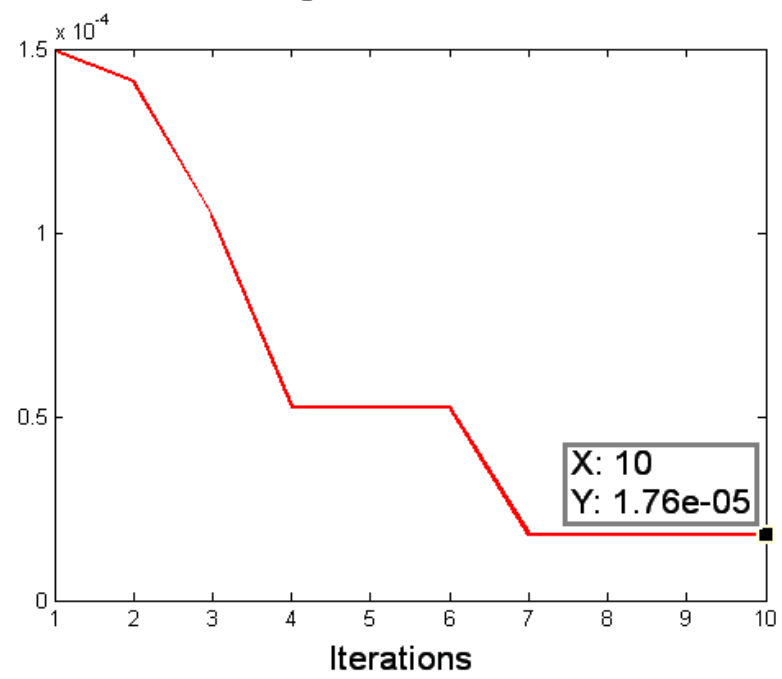

Fig 9: Average Power $=1.76 * 10^{-5}, \mathrm{VDD}=5 \mathrm{v}$, Temperature $=29.8766397972101^{\mathrm{c}}$

\subsection{Fuzzy-Genetic Algorithm (Fuzzy-GA)}

The fuzzy inference system is shown in Figure 10 [22]. The best average power is $3.14 * 10^{-5}$ Watt in Figure 11 .

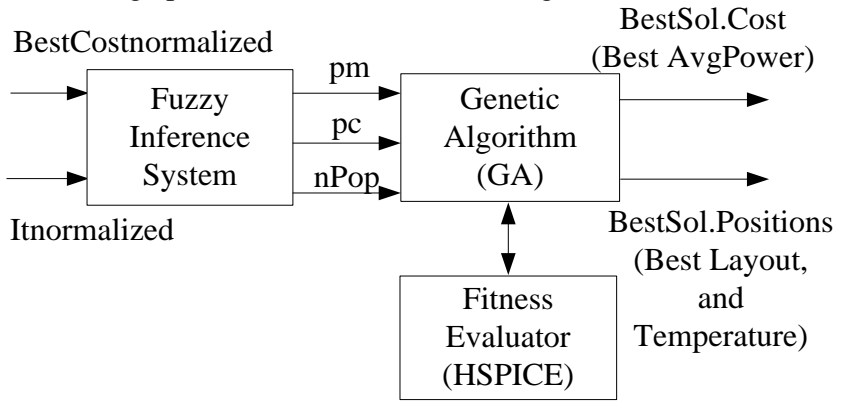

Fig 10: The Fuzzy Inference System in Fuzzy-GA

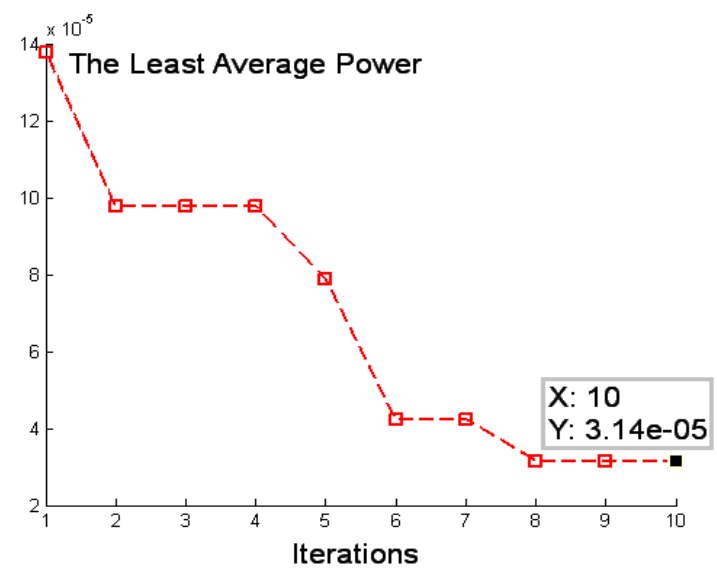

Fig 11: Average Power $=3.14 * 10^{-5}$ Watt, VDD=5v, Temperature $=25^{\mathrm{c}}$ based on Fuzzy-GA

The pseudo code of Fuzzy-GA is shown in Box 5.

Box 5. Fuzzy-GA Pseudo-Code which is used in Article
- Define the problem of 3 Stage Ring Oscillator, CostFunction=Objective Function ("in Box2"), nVar=6, VarMin: 0.2 for channel widths and 1 for temperature, VarMax: 2 for channel widths and 50 for temperature, MaxIt=10, nPop=4 (Population Size), pc=0.5 (Crossover Percentage), $\mathrm{pm}=0.5$ (Mutation Percentage)

- Generate Initial Population, Evaluate, Sort, and place them in the Best Solutions

- Do While iteration number (it) < Maximum Iteration (MaxIt)

- Calculate Selection Probabilities

- Select Parents indices and apply crossover and evaluate off springs, in Crossover Operator

- Select Parents indices and apply mutation and evaluate Mutant, in Mutation Operator

- Create Merged Population, sort population

- Update the best solution and the worst solution that ever found and Store Best Cost and Worst Cost

- Normalization of variables : itnormalized $=i t /$ MaxIt, and BestCostnormalized $=[$ WorstCost - BestCost(it) $] /$ WorstCost

- Read Fuzzy Inference System File and Fuzzy Rules (Fuzzy_GA_FIS.fis)

- Save the results (BestCost)

\section{- End Do}

- Plot Results

The optimum control of mutation percentage ("pm"), crossover percentage ("pc"), and the number of population ("nPop") is obtained by Fuzzy-GA rules that are illustrated in Table 5. The best solutions are shown in Table 6.

Table 5. The Fuzzy Rules in Fuzzy-GA

\begin{tabular}{|c|c|}
\hline If & Then \\
\hline Itnormalized is High and & pm is High and pc is Low \\
aestCostnormalized is High & anpop is High \\
\hline Itnormalized is High and & pm is Low and pc is High \\
BestCostnormalized is Low & and nPop is Low \\
\hline Itnormalized is Low and & pm is High and pc is Low \\
BestCostnormalized is High & and nPop is High \\
\hline Itnormalized is Medium and & All pm, pc, and nPop are \\
BestCostnormalized is Medium & Medium \\
\hline
\end{tabular}

Table 6. Channel Widths ("BestSol.Positions")

\begin{tabular}{|c|c|}
\hline $\mathbf{W 1}=W 3=W 5(\boldsymbol{\mu m})$ & $\mathbf{W} 2=W 4=W 6(\boldsymbol{\mu m})$ \\
\hline 0.486219988184535 & 1.34202178834180 \\
\hline
\end{tabular}

The values of channel widths that were shown in Table 6 remain unchanged as the input netlist file ("V3.sp") in HSPICE and the parameter of power supply is changed. The optimization is performed to find the best solution for the 
temperature and the best result is shown in Figure 12 .

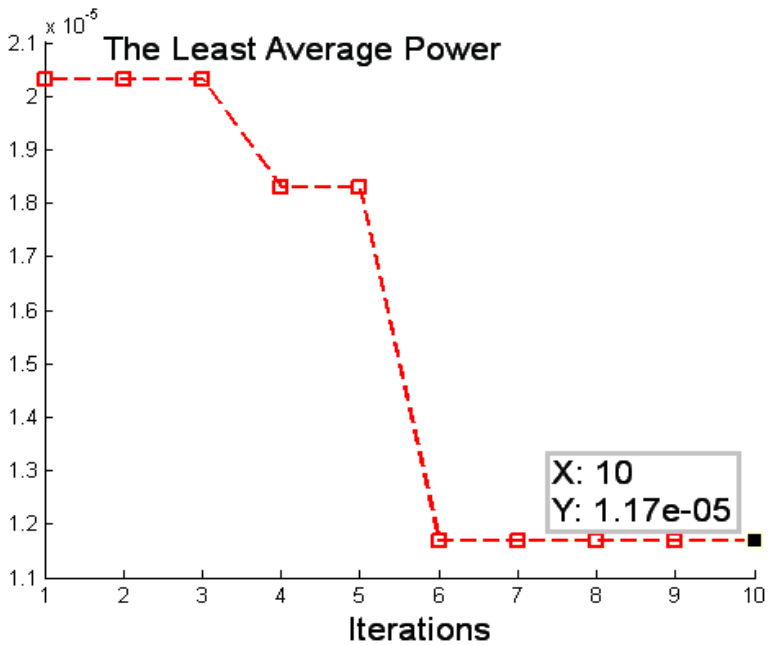

Fig 12: Average Power $=1.17 * 10^{-5}, \mathrm{VDD}=5 \mathrm{v}$, Temperature $=27.9075611137555^{\mathrm{C}}$

\section{CONCLUSIONS}

By implementation of meta heuristic algorithms such as Modified SFLA with cognitive behavior (MSFLA), FuzzyMSFLA, GA, and Fuzzy-GA the least average power is compared and it has been obtained for the specific values of the parameters such as temperature, NMOS channel widths, and PMOS channel widths. These parameters has effect on the average power consumption in 3 stage CMOS ring oscillator. In this article the least average power of $1.19 \mu \mathrm{w}$ is obtained based on Fuzzy-MSFLA in technology $\mathrm{L}=0.18 \mu \mathrm{m}$ and $\mathrm{V}_{\mathrm{DD}}=5 \mathrm{v}$. The comparisons are illustrated in Figure 13 and Table 7.

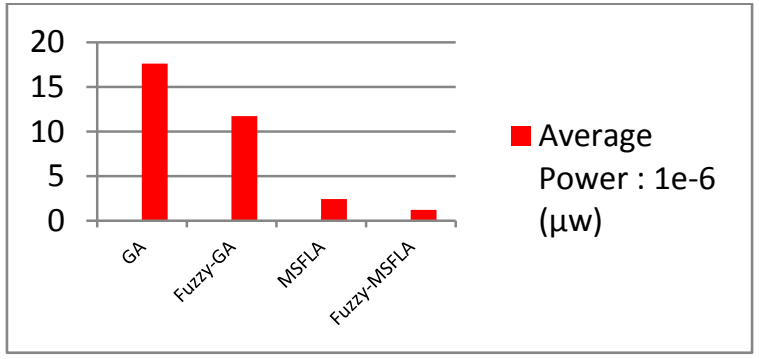

Fig 13: The Graphical Comparison

Also the Fuzzy-MSFLA and Fuzzy-GA showed the better results since the fuzzy rules increase the performance of the algorithm. The Fuzzy-MSFLA and Fuzzy-GA showed that the lower average power is resulted in lower temperature.

Table 7. The Comparative Results for Average Power in 3 Stage Ring Oscillator

\begin{tabular}{|c|c|c|c|c|}
\hline $\begin{array}{c}\text { Heuristic } \\
\text { Algorithms }\end{array}$ & $\begin{array}{c}\text { The } \\
\text { Least } \\
\text { Average } \\
\text { Power, } \\
\left(\mathbf{P}_{\text {avg }}\right)\end{array}$ & $\begin{array}{c}\text { Temperature } \\
(\text { Centigrade })\end{array}$ & $\begin{array}{c}\mathbf{W}_{\text {NMos }} \\
(\boldsymbol{\mu m})\end{array}$ & $\begin{array}{c}\mathbf{W}_{\text {PMos }} \\
(\boldsymbol{\mu m})\end{array}$ \\
\hline $\begin{array}{c}\text { Fuzzy- } \\
\text { MSFLA }\end{array}$ & $1.19 \mu \mathrm{w}$ & $\approx 29$ & 0.32 & 0.29 \\
\hline MSFLA & $2.4 \mu \mathrm{w}$ & $\approx 46$ & 0.63 & 1.66 \\
\hline GA & $17.6 \mu \mathrm{w}$ & $\approx 29$ & 0.64 & 1.5 \\
\hline Fuzzy-GA & $11.7 \mu \mathrm{w}$ & $\approx 27$ & 1.34 & 0.48 \\
\hline
\end{tabular}

\section{ACKNOWLEDGMENTS}

Dr. Khaliji provided me with SFLA code in MATLAB.

\section{REFERENCES}

[1] Michaelsen, J. A. 2012. Tutorial notes about "Ring Oscillators", INF4420 course material at the Department of Informatics, University of Oslo, Norway.

[2] Thakur, V., and Verma, V. 2013. "Low Power Consumption Differential Ring Oscillator", International Journal of Electronics and Communications Engineering, ISSN 0974-2166 Volume 6, Number 1,pp. 81-92.

[3] Razavi, B. 2010. "Design of Analog CMOS Integrated Circuit", Tata McGraw Hill pvt ltd, Edition 2002, Eighteenth reprint.

[4] Yao, Y. Z. 2006. "Comparative Study of Low Phase Noise Voltage Controlled Oscillator (VCOs) in CMOS Technology" M.Sc., Concordia University, Montreal, Quebec, Canada.

[5] Jovanovi'c, G., Stoj`cev, M., Stamenkovic, Z. 2010 A CMOS voltage controlled ring oscillatorwith improved frequency stability. Sci Publ State Univ Novi Pazar A Appl MathInform Mech; 2:1-9.

[6] Park, CH., Kim, O., Kim, B. 2001. A 1.8-GHz Selfcalibrated phase locked loop with precise I/Q matching. IEEE J Solid-State Circuits, 36:777-83.

[7] Sun, L., and Kwasniewski, TA., 2001. A 1.25-GHz 0.35$\mathrm{m}$ monolithic CMOS PLL based on a multiphase ring oscillator. IEEE J Solid-State Circuits, 36: 910-6.

[8] Zhang, X., Hu, X., Cui, G., Wang, Y., and Niu, Y.(2008, June). "An improved shuffled frog leaping algorithm with cognitive behavior", Intelligent Control and Automation, 2008, WCICA 2008, $7^{\text {th }}$ World Congress on (pp. 6197-6202). IEEE.

[9] Khaliji, M. 2014. "Theoretical and Practical training of Shuffled Frog Leaping Algorithm, SFLA", Researcher in Control Engineering, University of Tabriz, Iran, The training source of Artificial Intelligence in Iran; Tutorial website of www.matlabsite.com

[10] Docking, S. 2002. "Oscillation Frequency of a Ring Oscillator", A thesis of Master of Science, Electrical and Computer Engineering, University of Waterloo.

[11] Ranjith, J., and Muniraj, NJR. January 2013. "Novel Evolutionary Algorithm for ICA Processor for FPGA Implementation", International Journal of Soft Computing and Engineering (IJSCE), ISSN: 2231-2307, Vol-2,Issue-6.

[12] Zamani, M. S., Safaei, F., and Fathy, M. 2012. "Digital VLSI Design", Fifth Publication in Academic Book in Iran, ISBN: 978-964-92527-4-2.

[13] Ramazani, A., Biabani, S., and Hadidi, G. 2014. "CMOS ring oscillator with combined delay stages", International Journal of Electronics and Communications (AEU) 68 515-519, Elsevier.

[14] Fishchuk, I. I., Kadashchuk, A. K., Genoe, J., Ullah, M., Sitter, H., Singh, T. B., Sariciftci, N. S., and Bassler, H. 2010. "Temperature dependence of the charge carrier mobility in disordered organic semiconductors at large carrier concentrations", Physical Review B 81, 045202. 
[15] Duan, Q., Sorooshian, S., and Gupta, V. 1992. Effective and efficient global optimization for conceptual rainfallrunoff models. Water resources research, 28(4), 10151031.

[16] Duan, Q. Y., Gupta, V.K., \& Sorooshian, S. 1993. Shuffled complex evolution approach for effective and efficient global minimization. Journal of optimization theory and applications, 76(3), 501-521.

[17] Eusuff, M. M., \& Lansey, K. E. 2003. "Optimization of water distribution network design using the shuffled leaping algorithm. Journal of Water Resources Planning and Management, 129(3), 210-225.

[18] Eusuff, M., Lansey, K., and Pasha, F. 2006. Shuffled frog-leaping algorithm: a memetic meta-heuristic for discrete optimization. Engineering Optimization, 38(2), 129-154.

[19] Huynh, T. H. 2008, April 2008. "A modified shuffled frog leaping algorithm for optimal tuning of multivariable PID controllers." In Industrial Technology, , IEEE International Conference on (pp.1-6). IEEE.

[20] Farahani, M., Movahhed, S. B., and Ghaderi, S. F. (2010). "A hybrid meta-heuristic optimization algorithm based on SFLA", In proceedings of the $2^{\text {nd }}$ International Conference on Engineering Optimization (pp. 1-8).

[21] Ahdani, M. A., Shirjoposh, N. P., and Banimahd, R. (2010). "Three modified versions of differential evolution algorithm for continuous optimization" , Soft Computing, 15(4), 803-830.

[22] Keivanian, F., Mehrshad, N., and Zahiri, S. H. 2014. "Optimum Layout of Multiplexer with Minimal Average Power based on IWO, Fuzzy-IWO, GA, and Fuzzy GA", ACSIJ Advances in Computer Science : an International Journal, Vol. 3, Issue 5, No. 11, September 2014. 